\title{
FINAL STATE PROBLEM FOR THE NONLOCAL NONLINEAR SCHRÖDINGER EQUATION WITH DISSIPATIVE NONLINEARITY
}

\author{
MAMORU OKAMOTO AND KOTA URIYA
}

Abstract. We consider the asymptotic behavior of solutions to the nonlocal nonlinear Schrödinger equation with dissipative nonlinearity. We prove that there exists a solution which has different behavior from that of the typical cubic nonlinear Schrödinger equation.

Mathematics subject classification (2010): 35Q55.

Keywords and phrases: Nonlinear Schrödinger equation, asymptotic behavior, critical nonlinearity, dissipative nonlinearity.

\section{REFERENCES}

[1] M. J. Ablowitz And Z. H. Musslimani, Integrable nonlocal nonlinear Schrödinger equation, Phys. Rev. Lett. 110 (2013), no. 6-8.

[2] P. DeIFT AND X. ZHOU, Long-time asymptotics for solutions of the NLS equation with initial data in a weighted Sobolev space, Comm. Pure Appl. Math. 56 (2003), no. 8, 1029-1077.

[3] N. Hayashi, C. LI, AND P. I. NAUMKIn, On a system of nonlinear Schrödinger equations in 2D, Differential Integral Equations 24 (2011), no. 5-6, 417-434.

[4] N. Hayashi, C. Li, AND P. I. NAUMKin, Modified wave operator for a system of nonlinear Schrödinger equations in 2d, Comm. Partial Differential Equations 37 (2012), no. 6, 947-968.

[5] N. HAYASHI AND P. I. NAUMKIN, Asymptotics for large time of solutions to the nonlinear Schrödinger and Hartree equations, Amer. J. Math. 120 (1998), no. 2, 369-389.

[6] N. HAYASHI AND P. I. NAUMKIN, Domain and range of the modified wave operator for Schrödinger equations with a critical nonlinearity, Comm. Math. Phys. 267 (2006), no. 2, 477-492.

[7] N. Hayashi, P. I. Naumkin, A. Shimomura, and S. Tonegawa, Modified wave operators for nonlinear Schrödinger equations in one and two dimensions, Electron. J. Differential Equations 2004, No. $62,16 \mathrm{pp}$.

[8] N. Hayashi, P. I. Naumkin, And H. Sunagawa, On the Schrödinger equation with dissipative nonlinearities of derivative type, SIAM J. Math. Anal. 40 (2008), no. 1, 278-291.

[9] N. HAYAShI, H. WANG, AND P. I. NAUMKIn, Modified wave operators for nonlinear Schrödinger equations in lower order Sobolev spaces, J. Hyperbolic Differ. Equ. 8 (2011), no. 4, 759-775.

[10] J. Ieda, T. MiyaKaWa, And M. Wadati, Matter-Wave Solitons in an F 1 Spinor Bose-Einstein Condensate, J. Phys. Soc. Jpn. 73 (2004), 2996-3007.

[11] M. IFRIM AND D. TATARU, Global bounds for the cubic nonlinear Schrödinger equation (NLS) in one space dimension, Nonlinearity 28 (2015), no. 8, 2661-2675.

[12] J. KATO AND F. PUSATERI, A new proof of long-range scattering for critical nonlinear Schrödinger equations, Differential Integral Equations 24 (2011), no. 9-10, 923-940.

[13] Y. Nakamura, A. Shimomura, And S. Tonegawa, Global existence and asymptotic behavior of solutions to some nonlinear systems of Schrödinger equations, J. Math. Sci. Univ. Tokyo 22 (2015), no. 3, 771-792.

[14] T. OGAWA AND K. URIYA, Final state problem for a quadratic nonlinear Schrödinger system in two space dimensions with mass resonance, J. Differ. Equ. 258 (2015), 483-503.

[15] T. OZAWA, Long range scattering for nonlinear Schrödinger equations in one space dimension, Comm. Math. Phys. 139 (1991), no. 3, 479-493. 
[16] A. Shimomura AND S. Tonegawa, Long-range scattering for nonlinear Schrödinger equations in one and two space dimensions, Differential Integral Equations 17 (2004), no. 1-2, 127-150.

[17] A. ShimomURA, Asymptotic behavior of solutions for Schrödinger equations with dissipative nonlinearities, Comm. Partial Differential Equations 31 (2006), no. 7-9, 1407-1423.

[18] H. SunAGAWA, Large time behavior of solutions to the Klein-Gordon equation with nonlinear dissipative terms, J. Math. Soc. Japan 58 (2006), no. 2, 379-400.

[19] Y. TsutsumI, $L^{2}$-solutions for nonlinear Schrödinger equations and nonlinear groups, Funkcial. Ekvac. 30 (1987), no. 1, 115-125.

[20] K. URIYA, Final state problem for systems of cubic nonlinear Schrödinger equations in one dimension, Ann. Henri Poincaré 18 (2017), no. 7, 2523-2542. 